\title{
8
}
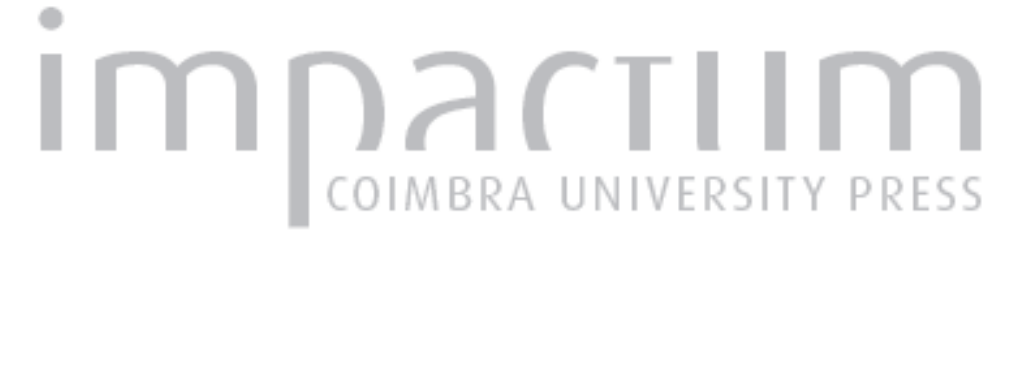

\section{Un problème d'environnement rural: La dangerosité du loup en France. Du Moyen Âge au xxe siècle}

Autor(es): Moriceau, Jean-Marc Publicado por: $\begin{aligned} & \text { Instituto de História Económica e Social, Faculdade de Letras da } \\ & \text { Universidade de Coimbra }\end{aligned}$

URL

persistente:

DOI: $\quad$ DOI:http://dx.doi.org/10.14195/0870-4147_41_1

Accessed : $\quad$ 26-Apr-2023 13:15:48

A navegação consulta e descarregamento dos títulos inseridos nas Bibliotecas Digitais UC Digitalis, UC Pombalina e UC Impactum, pressupõem a aceitação plena e sem reservas dos Termos e Condições de Uso destas Bibliotecas Digitais, disponíveis em https://digitalis.uc.pt/pt-pt/termos.

Conforme exposto nos referidos Termos e Condições de Uso, o descarregamento de títulos de acesso restrito requer uma licença válida de autorização devendo o utilizador aceder ao(s) documento(s) a partir de um endereço de IP da instituição detentora da supramencionada licença.

Ao utilizador é apenas permitido o descarregamento para uso pessoal, pelo que o emprego do(s) título(s) descarregado(s) para outro fim, designadamente comercial, carece de autorização do respetivo autor ou editor da obra.

Na medida em que todas as obras da UC Digitalis se encontram protegidas pelo Código do Direito de Autor e Direitos Conexos e demais legislação aplicável, toda a cópia, parcial ou total, deste documento, nos casos em que é legalmente admitida, deverá conter ou fazer-se acompanhar por este aviso. 



\title{
Un problème d'environnement rural: La dangerosité du loup en France. Du Moyen Âge au $\mathrm{Xx}^{\mathrm{e}}$ siècle*
}

\author{
JEAN-MARC Moriceau \\ Université de Caen Basse-Normandie \\ jean-marc.moriceau@unicaen.fr
}

\section{Resumé:}

Alors que des oppositions s'élèvent entre partisans et adversaires du loup, la question de la dangerosité du prédateur sur l'homme laisse perplexe. À quoi correspond l'image négative du prédateur? Quelle contribution l'historien peut-il apporter selon ses sources et ses méthodes de travail? À l'issue d'une collecte de 5400 cas d'attaques sur l'homme, survenues surtout entre 1580 et 1880 , on saisit la chronologie et la distribution spatiale du risque. Avérées mais anciennes, statistiquement faibles mais anthropologiquement insupportables, ces agressions opposent en fait les loups prédateurs, qui considèrent l'homme comme une proie occasionnelle, et les loups enragés qui frappent la population sans discernement. Récurrentes dans un environnement peu sécurisé, les attaques des premiers ont donné corps à la croyance aux «Bêtes féroces», au cours d'épisodes dramatiques (1594-1600, 1693-1694, 1746-1750, 1764-1767, 1814-1819). Davantage circonscrites dans le temps et dans l'espace, les attaques des seconds ont semé une psychose d'autant plus durable qu'elles annonçaient une maladie incurable jusqu'au dernier tiers du XIXe siècle. La confusion entre ces deux ordres de réalité a forgé, pour Canis Lupus, une image négative qui se délite depuis qu'elle s'est éloignée pour le cas de la France-de toute matérialité.

\section{Mots-clé:}

Loup prédateur; Loup enragé; Bête féroce; Méthodologie historique; Dangerosité animale; Risque anthropophagique.
Abstract:

In a context of stark opposition between defenders and opponents of the wolf, the extent to which this particular predator is dangerous for humans is still an open question. What lies beneath the negative perception of the wolf ? What should be the historian's contribution, based on historical sources and historical methods? Thanks to a database of 5,400 cases of wolf attacks on humans having occurred in France between 1580 and 1880, it is now possible to grasp the chronological evolution and spatial evolution of wolf-related risks. Undeniably real, though mostly belonging to a remote past, and statistically rare, though anthropologically intolerable, these attacks point to two opposite groups, predatory wolves ocasionally preying on humans on one hand, rabid wolves striking indiscriminately a whole population on the other. The attacks of the first kind occured regularly in an unsafe environment, and gave rise to tales of «Ferocious Beasts» in times of dramatic crisis (1594-1600, 1691-1694, 1746-1750, 1764-1767, 1814-1819). The second kind was spatially and chronologically less widespread, but sowed the seeds of mass hysteria, all the more persistent since the underlying sickness it forebode remained without a cure before the last third of the XIXth century. The confusion between these two different realities gave Canis Lupus its negative aura, significantly dimmed recently however since it has entirely lost its material basis, at least in France.

Keywords:

Predatory wolf; Rabid wolf; Ferocious beast; Historical methodology; Animal dangerosity; Anthropophagous risk. 


\begin{abstract}
«Marie, fille âgée d'environ 7 ans, de Jacques Prudent et de Tiennette Maroyer sa première femme, a été prise par un loup à la porte de sa maison, et dévorée dans un champ. On n'a trouvé que la tête, un bras et l'estomac et rien autre chose. Ces tristes restes ont été inhumés le lendemain cinq d'octobre au cimetière de cette église en présence de toute ma paroisse qui était rassemblée pour entendre la messe du saint Dimanche.»
\end{abstract}

Registres paroissiaux de La Chapelle-Thècle (Saône-et-Loire), 8 octobre 1749 (Arch. dép. Saône-et-Loire, état civil en ligne, La Chapelle-Thècle, Baptêmes, mariages et sépultures, 1743-1752, vue 66).

Des siècles duRAnt le loup a incarné le premier ennemi de l'homme'. Contre Canis lupus la France, mais aussi la plupart des États européens, ont mobilisé des ressources importantes pour contenir puis éradiquer celui qu'ils ont longtemps considéré comme le pire des animaux «nuisibles». Sa destruction a commandé presque partout un financement spécifique dont les modalités ont été très voisines. Qu'il s'agisse de la paga del llop qui figure dans les coutumes de Catalogne depuis le XIV siècle, des différents premi per gli occisori di lupi, qui jalonnent les statuts communaux en Italie du Nord comme en Suisse du XV $\mathrm{Xv}^{\mathrm{e}}$ au XIX ${ }^{\mathrm{e}}$ siècle, des primes en escalins établies en Flandre ou en Brabant depuis au moins 1397 ou de celles en maravédis que l'on délivre dans la région de Murcie dès le XIv $v^{\mathrm{e}}$ siècle ou enfin des tallas de fieras accordées dans de nombreux villages de la principauté des Asturies au XVIII ${ }^{\mathrm{e}}$ siècle, la chasse au loup a été encouragée partout ${ }^{2}$.

En France, le canidé a été exterminé entre 1882 et 1930. La dernière prime accordée pour la destruction d'un loup qui s'était jeté sur un être humain remonte à 1896 et la dernière attaque supposée sur l'homme à 1918. Le dernier loup «primé» l'a été dans le département du Cantal en 1927. Mais, depuis quelques décennies, il n'y avait plus que des loups erratiques. Jusqu'à son retour dans à l'ouest des Alpes depuis l'Italie en 1992, le loup n'a plus été l'hôte du territoire français. Au cours de cette longue absence - le temps de deux à trois générations pour les hommes - notre représentation négative de Canis lupus,

1 Jean Trinquier, «Vivre avec le loup dans les campagnes de l'Occident romain», Le loup en Europe du Moyen Âge à nos jours. Études réunies par Fabrice Guizard-Duchamp, Presses universitaires de Valenciennes, 2009, pp. 1-39.

2 Albert Manent, El llop a Catalunya. Memoria, llegenda i historia, Lleida, 2004, pp. 152-157 (d'après notamment les travaux d'Albert Curto sur le diocèse de Tortosa); Mario Comincini (éd.), L'Uomo e la «bestia antropofaga», Milan, Unicopli, 2002, pp. 148-160; Adrien de Melotte de Lavaux, Le loup dans la légende et dans l'histoire, Liège, 1938, p. 47; Juan Pablo Torrente, «La chasse aux grands carnivores dans les Asturies au XVIII ${ }^{e}$ siècle. Les papeletas de fieras», Histoire et Sociétés Rurales, 8, 2 ${ }^{\mathrm{e}}$ semestre 1997, pp. 163-186; Robert Delort, Les animaux ont une histoire, Paris, 1984, pp. 326-327. 
traditionnellement nourrie de faits-divers tragiques s'est détachée peu à peu de toute réalité effective. Que reste-t-il dans les mémoires d'un «carnage» comme celui de 1749, qu'on vient de citer en exergue, au cours duquel une fillette de 7 ans avait été dévorée par un loup jusqu'aux os? Sans doute rien, malgré le souci d'un curé de l'actuel département de Saône-et-Loire d'en donner «avis à la postérité». En France, à la différence de quelques autres régions du monde comme l'Inde, la Turquie ou la Russie, la dangerosité du loup appartient à un passé déjà éloigné.

Par ailleurs, la transformation de la place de l'homme et de l'animal sauvage a conforté un changement de perception, engagé après la Seconde Guerre mondiale. La gestion actuelle de l'espace rural et de l'environnement limite les occasions conflictuelles entre l'homme et l'animal. Aujourd'hui, le loup ne pose de problèmes effectifs qu'à un secteur restreint de l'élevage, cantonné encore à quelques départements de la France du Sud-Est, tandis que son image est devenue très positive auprès d'un large public. En quelques décennies, le loup est passé - dans une partie de l'opinion - du statut d'animal honni, bête féroce qui «mange les hommes», à celui de «super-prédateur» protégé. Le renversement de perspective répond à un souci récent - la protection de la biodiversité -, alors que les questions d'écologie sont passées au premier plan. Cependant, le débat est loin d'être tranché et les tensions régionales sont vives avec le retour de l'animal sur le versant français des Alpes.

\section{L'historien engagé: dépassionner le débat}

Ce débat actuel autour du loup remet en cause l'héritage culturel sur lequel reposait notre représentation de l'animal. La juste appréciation des relations entre le carnivore et l'homme s'est brouillée et, souvent s'impose un postulat, que confirment les observations actuelles de la plupart des biologistes: «le loup n'attaque pas l'homme». De là, et sans prendre la peine d'en vérifier le bien-fondé, se répand l'idée commune que le loup n'a jamais attaqué d'être humain, en dehors de cas d'animaux enragés, sur lesquels on ne s'étend guère. Ce mode de raisonnement par analogie fait litière du passé. Chez certains écologistes, il sert d'arme pour assurer la protection du prédateur, jusqu'à susciter un second postulat: l'intérêt de la biodiversité, et en particulier de la préservation de Canis Lupus, déconseille de lever le voile sur des éléments qui lui seraient défavorables. Chez quelques gestionnaires, en particulier dans les Parcs nationaux de régions directement concernées par le retour du loup, une certaine réserve s'impose pour des raisons «politiques». Le constat laisse l'observateur perplexe. La cause du retour du loup est-elle nécessairement 
associée à un tri dans les informations dont nous disposons sur ses rapports avec l'homme? Un fait qui longtemps allait de soi en est venu à devenir une question délicate, parfois taboue: à quelles réalités effectives correspond l'image du loup «mangeur d'hommes» ${ }^{3}$ ?

Dans un pays comme la France, qui a accueilli jusqu'au XIX e siècle l'une des populations de canidés sauvages les plus importantes en Europe - 10 à 15000 loups à la fin du XVIII ${ }^{\mathrm{e}}$ siècle -, et qui a connu aussi l'une des densités de population les plus fortes dans l'espace rural, la réponse que nous apportons ici est étayée sur de multiples sources d'archives. Dans ce contexte, nous nous assignons ici deux grands objectifs:

- Établir la place de l'historien dans un débat public «informé», pour reprendre les propos que tenait John Linnell dans une enquête internationale sur la peur du loup ${ }^{4}$, en exposant sa mission, ses sources et ses méthodes.

- Présenter les bases documentaires et les premiers résultats d'une enquête nationale sur les attaques du loup sur l'homme pour comprendre l'impact mémoriel du canidé sauvage en France et en faciliter la gestion.

\section{L'historien dans un débat public}

\section{La posture du chercheur}

Au préalable, il importe de clarifier l'objet de la discussion, souvent déformé par les appréciations d'auteurs extérieurs à la science historique. Car en la matière, bien des interventions qui se réfèrent au passé s'en tiennent à des idées reçues, quand il ne s'agit pas de simples partis pris émanant de certains secteurs de l'opinion publique, qui expriment leur position idéologique sur la place du loup aujourd'hui, qu'ils soient favorables («lycophiles») ou hostiles («lycophobes») au canidé. Le souci de cautionner un point de vue ou de réfuter son contraire conduit à instrumentaliser le passé. L'apparente accessibilité au public de la documentation historique multiplie les risques d'anachronisme, de déformation ou de surinterprétation. Entre le XVII et le $\mathrm{XXI}^{\mathrm{e}}$ siècle des mots

${ }^{3}$ Cette contribution complète et met à jour celle que l'auteur a déjà donnée à l'ouvrage collectif: Repenser le sauvage grâce au retour du loup. Les sciences humaines interpellées, éd. Jean-Marc Moriceau et Philippe Madeline, Caen, Presses universitaires, «Bibliothèque du Pôle Rural, 2», 2010, pp. 41-74 (Jean-Marc Moriceau, «La dangerosité du loup sur l'homme. Une enquête à l'échelle de la France $\left(\mathrm{XvI}^{\mathrm{e}}-\mathrm{Xx}^{\mathrm{e}}\right.$ siècle)»). Le lecteur intéressé y trouvera toutes les références de sources qui n'apparaissent pas ici ainsi que des essais d'évaluation des risques causés par les attaques de Canis lupus sur l'homme.

4 John D. C. Linnel et al., The Fear of Wolves. A Review of Wolf attacks on Humans, Trondheim Norsk Institutt for Naturforskning, 2002, p. 7. 
restés identiques ont changé de sens et les réalités auxquelles ils renvoient ont pu varier. Les bouleversements de l'économie, des techniques et de la société ont exercé un impact indéniable sur l'environnement. Aussi importe-t-il de disposer d'une bonne appréciation du contexte spatio-temporel. En outre, le progrès de notre connaissance commande une vision large des faits, qui passe par des mesures statistiques et des changements d'échelle d'analyse. C'est à ce prix que l'historien entend apporter une contribution constructive au débat général. Dans cette perspective, il est conduit, plus que jamais, à expliciter les procédures de validation de son travail de chercheur:

- Critiquer: de quels témoignages dispose-t-on et quelle en est la validité?

- Distinguer: quel type d'agression selon qu'il s'agit d'animaux sains (prédateurs) ou enragés?

- Mesurer et localiser: combien de cas enregistrés? suivant quelle évolution chronologique et quelle répartition géographique?

- Identifier: comment reconnaître les agresseurs et quelle en fut la perception culturelle?

- Contextualiser: quel fut l'impact démographique et sociologique des attaques?

\section{Un chantier en plusieurs étapes}

Dans un premier temps, de 2003 à 2007, il fallait rassembler les travaux existants: plusieurs centaines de références dispersées sur deux siècles et demi de publications. De l'examen de ce vaste corpus bibliographique, s'est dégagé un cadre d'analyse inter-régional. Pour assurer une couverture nationale à l'échelle de la France, une enquête a été menée auprès des 95 dépôts d'archives départementales de la France métropolitaine. L'excellent taux de réponse $(90 \%)$ obtenu a ouvert de nombreuses pistes de recherche en vue d'une synthèse nationale. Toutefois, en dehors de ce travail nécessaire, la progression scientifique requérait un apport quantitatif. Dans ce dessein, deux tableurs ont été constitués à partir des attaques de loups déjà mentionnées dans la bibliographie puis à travers des recherches ponctuelles en archives:

- un premier rassemble les victimes de loups enragés qui ont attaqué l'homme sous l'emprise de la maladie;

- un second regroupe les victimes de loups sains, qui ont manifesté, à l'occasion, un comportement anthropophage.

Mais l'ampleur de la tâche dépassait les capacités d'un individu. Il fallait passer à l'échelle de l'enquête collective. Dans les années 1990, Jacques 
Dupâquier avait été confronté au même défi pour reconstituer la mobilité sociale des Français de la fin du XVIII à la fin du Xx ${ }^{\mathrm{e}}$ siècle. Pour disposer d'une base d'information représentative de l'ensemble des départements français, il avait mobilisé les généalogistes. Ainsi son enquête - l'enquête «TRA»d'après les trois premières lettres des patronymes sélectionnés -, avait pu déboucher sur un corpus national de 3000 familles ${ }^{5}$. Sur le même principe, j'ai fait appel aux associations de généalogistes, réparties sur toute le France. La structuration associative de ces milliers de chercheurs amateurs et la mise en ligne de leurs résultats ont accéléré la collecte des données. De fil en aiguille, les appels à la recherche étant relayés d'une région à l'autre, plus de 3000 attaques ont été retrouvées. Pour chacune, la localisation des agressions dans le temps et dans l'espace, l'identification et la désignation des agresseurs, l'âge, le sexe et la condition sociale des victimes ouvraient la voie à une étude sociale autant qu'environnementale. Examinés à plusieurs échelles, du local au national, de la séquence annuelle à l'évolution décennale et séculaire, ces drames répétés éclairent les rapports entre l'homme et son environnement dans la gestion de son territoire. Fort de cette base statistique, un premier bilan a été proposé au public en juin 2007: Histoire du méchant loup. 3000 attaques sur l'homme en France $\left(X V^{e}-X X^{e}\right.$ siècle). Le lecteur y trouvera les analyses et les développements particuliers qui ne sauraient apparaître ici ${ }^{6}$.

En juillet 2008, un changement d'échelle a permis d'intégrer à l'étude les ravages qui frappèrent le Gévaudan et l'Auvergne de 1764 à 1767. Ceux-ci apportent un éclairage nouveau sur l'action des grands prédateurs dans un environnement particulier et sur une société aux portes de la misère, dans un pays oublié qui sinon n'aurait pas attiré l'attention. Par rapport à la littérature et au cinéma qui en ont tiré profit sans compter, cette nouvelle étape éditoriale - La Bête du Gévaudan (1764-1767) - prend aussi l'animal comme un révélateur du passé ${ }^{7}$. Cette seconde contribution met en évidence la spécificité du terme de «bête» comme mode de désignation des agressions sur l'homme et la parenté de ces épisodes hors normes à travers l'histoire. Une comparaison avec des cas d'attaques en série retrouvés à l'étranger, et notamment par Hans Kruuk pour la Hollande et la Biélorussie aux XIX ${ }^{\mathrm{e}}$ et $\mathrm{XX}^{\mathrm{e}}$ siècles, est venue atténuer la singularité

5 Jacques Dupâquier et Denis Kessler, La Société française au XIX siècle. Tradition, transition, transformations, Paris, Fayard, 1992.

6 Jean-Marc Moriceau, Histoire du méchant loup. 3000 attaques sur l'homme en France ( $X V^{e}-X X^{e}$ siècles), Paris, Fayard, 2007, ouvrage complété et réédité en 2008.

7 Jean-Marc Moriceau, La Bête du Gévaudan (1764-1767), Paris, Larousse, 2008. 
des exemples que j'avais déjà retrouvés ${ }^{8}$. Le cas français n'est donc pas isolé et les études déjà réalisées ponctuellement pour l'Italie - à commencer par celle de l'archiviste Mario Comincini pour l'Italie - et du naturaliste José Antonio Valverde pour l'Espagne le soulignent. Mais, même si certaines méthodes sont discutables - notamment dans la compilation effectuée par Will Graves sur la Russie -, elles n'ont guère trouvé d'écho dans le grand public ${ }^{9}$.

En France, le chantier est donc resté ouvert, en tirant parti des réactions d'un public inédit pour l'historien, qui disposait sur internet de forums de discussion actifs ${ }^{10}$. Sur la toile, la sortie en 2007 de l'Histoire du méchant loup a suscité des débats auprès des milieux sensibilisés à l'égard de l'animal sauvage et peu au fait de la recherche historique. Pour répondre aux interrogations des internautes une mise au point a paru nécessaire ${ }^{11}$. Bien qu'on les ait déjà largement traités, il a fallu revenir sur quelques points importants: la différence de comportement entre loups prédateurs et loups enragés; la fiabilité du témoignage des curés d'Ancien Régime; l'importance et la variété des autres sources documentaires qui faisaient état des attaques de loups; le sens du terme de «bête», utilisé lors des séquences d'attaques les plus traumatisantes. En dehors de ces simples rappels d'autres points ont conduit à plus de précision: le rôle des chiens errants

8 Hans Kruuk, Chasseurs et chassés. Relations entre l'homme et les grands prédateurs, Paris, Delachaux et Niestlé, 2005, 224 p.

9 Mario Cominchini (dir.), L'Uomo e la «bestia antropofaga». Storia del lupo nell'Italia settentrionale dal XV al XIX secole, Milano, Unicopli, 2002, 337 p.; José Antonio Valverde, Los lobos de Morla, Al Andalus Ediciones, 2001,550 p.; Will Graves, Wolves in Russia. Anxiety through the Ages, Calgary, Detselig, 2007, 223 p. (en particulier le chap. 6: «Wolf attacks on Humans»).

${ }^{10}$ Parmi les principaux sites: www.citedurable.com, «Une histoire du méchant loup» (19 mai 2007) - www.loup.org, «Histoire du méchant loup. Quelle contribution au débat sur les grands prédateurs? - ferus.org, «Histoire du méchant loup de J.M. Moriceau: vos réactions» (4 janvier 2008) - www.agrobiosciences.org, «Le loup à la fois révélateur de l'histoire des hommes et de l'histoire de la ruralité» (mai 2008) - loup.fne.asso.fr, «L'histoire et le grand méchant homme» - www.rue89.com, «Fallait-il avoir peur du grand méchant loup?» (27 décembre 2007) - blogs. laprovence.com, «Qui a peur du loup?» (12 janvier 2008) - la-meute.org, «Histoire du méchant loup. Quelle contribution au débat sur les grands prédateurs?» (21 septembre 2007) - www. planet.fr, «La chasse aux loups est ouverte» (15 octobre 2009) - fabrice-nicolino.com, «Un loup vraiment anthropophage» (30 août 2007) - www.legrandcharnier.blogs.sfr.fr, «Le Grand charnier en Lozère» (26 août 2009).

${ }^{11}$ Devant la nature de certaines réactions comme celle de Jean-Pierre Raffin («Quelques réflexions sur l'ouvrage de J-M Moriceau») une intervention sur internet a paru utile: Jean-Marc Moriceau, «Réponses à diverses critiques sur l'Histoire du méchant loup: Comment passer d'une discussion assez vaine à un débat un peu constructif?», www.loup.org, «Histoire du méchant loup. Quelle contribution au débat sur les grands prédateurs», 4 janvier 2008. 
et d'éventuels hybrides de chiens et de loups; la réalité de faits comparables en dehors de l'Hexagone. En même temps la base statistique a été quelque peu complétée (3 272 agressions au lieu de 3 069). Ces apports complémentaires ont été présentés au public dans une seconde édition de l'ouvrage en $2008^{12}$.

Cependant, une question était restée en suspens: le procédé quantitatif qui vise à ériger en séries statistiques un agrégat de faits-divers dont l'impact réel fut d'abord strictement localisé. Sur ce point l'éclairage qu'apporte le sociologue Antoine Doré en 2010, dans un ouvrage collectif et interdisciplinaire qui ouvre une nouvelle étape dans cette enquête, souligne que toute conclusion scientifique reste tributaire de sa mise en perspective ${ }^{13}$. Le chantier n'est donc pas fermé. Il prend place parmi d'autres réflexions sur la place du loup aujourd'hui comme dans le passé. Il aimante une recherche collective et pluridisciplinaire qui vient l'élargir. Il s'alimente des découvertes des uns et des autres, qui complètent les données déjà enregistrées: au 17 septembre 2010, on atteint presque 5400 attaques sur l'homme.

\section{À travers cartes et chiffres: 5400 victimes}

En accumulant les données de toutes origines qui indiquent des attaques dans nos sources ${ }^{14}$, on aboutit à un total provisoire de 5379 cas au 17 septembre 2010. L'ensemble constitue une base statistique indicative déjà importante mais dont l'homogénéité n'est pas parfaite. Elle comprend les attaques de loups prédateurs et celles des loups enragés dont les comportements sont fort différents. Il a donc fallu constituer deux corpus distincts, qui regroupent des effectifs désormais comparables mais sur des bases documentaires fort différentes, presque inverse dans leur structuration. ${ }^{15}$

${ }^{12}$ Jean-Marc Moriceau, Histoire du méchant loup, éd. corrigée et complétée, Paris, Fayard, 2008, cf en particulier l'avant-propos, pp. I-VI.

13 Antoine Doré, «L'histoire dans les méandres des publics: quand les «méchants loups» ressurgissent du passé», in Jean-Marc Moriceau et Philippe Madeline, éd., Repenser le sauvage grâce au retour du loup. Les sciences humaines interpellées, Caen, Presses universitaires de Caen, «Bibliothèque du Pôle rural, 2», 2010, pp. 75-90.

${ }^{14}$ Sur les sources documentaires et leur utilisation, cf Jean-Marc Moriceau, «La dangerosité du loup sur l'homme», in Repenser le sauvage grâce au retour du loup, 2010, pp. 41-74.

15 Jean-Marc Moriceau, L'Homme contre le loup. Une guerre de deux mille ans, Paris, Fayard, 2001, 408 p. 


\section{Deux bases de données complémentaires}

\begin{tabular}{|c|c|c|}
\hline \multicolumn{3}{|c|}{ Loups prédateurs et loups enragés (5 376 cas) } \\
\hline & Victimes de loups prédateurs & Victimes de loups enragés \\
\hline Données individualisées & $1714(66,8 \%)$ & $892(31,7 \%)$ \\
\hline (dont actes de décès) & $1626(63,3 \%)$ & $352(12,5 \%)$ \\
\hline Données collectives & $852(33,2 \%)$ & $1921(68,3 \%)$ \\
\hline Ensemble des victimes & 2566 & 2813 \\
\hline
\end{tabular}

Pour les loups prédateurs, non enragés, qui sélectionnent leurs victimes comme autant de proies potentielles, les attaques sont presque toujours individuelles. Pour les deux tiers des données, nous connaissons directement le nom de la victime. De fait, l'essentiel de nos informations repose sur les actes de décès qui ne concernent à chaque fois qu'une victime. En revanche, les données relatives aux simples blessés - il s'agit surtout des enquêtes administratives - sont rares. Les attaques de loups prédateurs sous-évaluent les réalités en raison des lacunes dans l'enregistrement individuel des décès et de la grande difficulté à identifier les blessés.

En revanche pour les attaques de loups enragés, qui se jettent sur tous les êtres vivants qu'ils rencontrent, du fait de leur maladie, les données ne sont individualisées que dans moins d'un tiers des cas et les actes de décès ne constituent qu'une minorité des données, à peine un huitième de l'échantillon. Bien des victimes sont parties mourir ailleurs et souvent longtemps après - la période d'incubation de la rage, très forte entre 15 jours et trois mois pouvant aller jusqu'à plus de un an. En revanche nos données sont surtout globalisées. Les documents - notations de curés, d'administrateurs ou de médecins fournissent souvent un chiffre total des victimes qui n'opère pas toujours de distinction entre celles qui sont mortes de la rage de celles qui semblent y avoir échappé. Une même attaque a pu produire un nombre de victimes très variable (de une à plusieurs dizaines). Les attaques de loups enragés présentent un caractère sériel qui les différencie de celles des loups mangeurs d'hommes. Pour cette raison, elles sont plus visibles dans la documentation mais l'information qu'elles procurent est souvent plus approximative.

Lors de la Révolution française, la centralisation des primes accordées pour détruire les loups facilite le repérage des attaques de loups enragés. Or ces séries documentaires viennent seulement d'être inventoriées pour les besoins de l'enquête ${ }^{16}$. C'est la raison pour laquelle, la proportion entre les deux types

${ }^{16}$ À cet égard je tiens à remercier Vanessa Pouteau, étudiante en master d'histoire à l'université de Caen, qui s'est livré avec beaucoup de soin aux dépouillements dans la sous-série F10 des Archives nationales. 
de victimes a évolué depuis le premier bilan réalisé avec l'Histoire du méchant loup en 2007: les victimes de loups enragés sont passé d'un gros tiers à plus de la moitié de l'ensemble. En poursuivant l'enquête patiemment dans les actes de sépulture - mais l'opération consiste souvent à rechercher une aiguille dans une botte de foin! - il a fort à parier que, pour les époques plus anciennes, la balance entre les deux types de victimes pourrait bien revenir un peu en arrière. Cependant, quel que soit le type d'agression, les données rassemblées offrent des points de repère dans le temps et dans l'espace qui aimantent la progression de la recherche.

Elles ont été entrées dans deux tableurs excel qui comportent un large éventail de rubriques pour assurer ensuite des traitements statistiques multivariés sous forme de tableaux croisés dynamiques. Pour les victimes de loups non suspectés de rage l'éventail des champs d'analyse comprend 25 rubriques: année (calendrier grégorien), période (1421-1660, 1661-1715, 1716-1750, 1751-1830 et 1831-1918), jour de l'accident (parfois différent du jour de la déclaration), mois, paroisse ou commune ancienne, commune actuelle, code INSEE, département, référence d'archive, âge retrouvé (et calculé à partir de l'acte de baptême), âge déclaré (dans l'acte de sépulture), âge retenu (qui intègre progressivement les âges retrouvés en complément des âges déclarés), sexe de la victime (féminin, masculin ou non précisé), identification de l'événement (description précise qui peut imposer une transcription complète de l'acte), prénom de la victime, nom de la victime, activité ou statut socio-professionnel de la victime ou de ses parents s'il s'agit d'un enfant, désignation de l'agresseur («bête» en forme de loup, «bête féroce», «bête carnassière», «loup féroce», «loup carnassier», «loup cervier», «loup ravissant», «loup vorace», «mauvais loup», etc.), nombre d'agresseurs (quand il est connu), déroulement de l'attaque (étranglement, effet de surprise, capture, transport), identification de la prédation (consommation de tout ou partie de la victime), restes découverts et inhumés (ossements, tête, entrailles, parties de corps), localisation dans la journée (heure approximative) et dans l'espace (chemin, village, champ, pré, lieu-dit, parcelle, section), circonstance de l'accident (en allant abreuver du bétail, en jouant dans la cour, en participant à la moisson, en gardant les vaches, etc.). L'abondance des champs d'analyse est à la mesure de la richesse de l'information mais il est tributaire des lacunes documentaires et du temps nécessaire à la mise en œuvre et à la vérification des données.

Le second corpus, réservé aux victimes des loups enragés est pour l'instant moins riche, en raison de l'abondance des données à caractère collectif qui ne précisent pas l'identité des victimes et rendraient vaine l'extension des rubriques. Il ne comporte donc que 15 champs d'analyse: année, jour, mois, 
attaque (une attaque pouvant générer plusieurs victimes), lieu (commune actuelle), département, prénom puis nom de la victime, sexe, âge, identification de l'agresseur (presque toujours «loup enragé»), description de l'événement, commentaire, délai entre l'attaque et la mort de la victime en cas de rage, catégorie ou statut socio-professionnel, source d'archive.

\section{Les agressions sur l’homme: une répartition géographique}

Par rapport aux premiers résultats publiés en 2007 (sur la base de 3058 cas) la répartition géographique d'ensemble des attaques (loups prédateurs + loups enragés) à laquelle on parvient avec près de 5400 cas n'a guère varié, en dépit de l'accroissement des effectifs. En dehors de la remontée d'un département comme le Maine-et-Loire (due aux terribles attaques de loups enragés de 1714 qui firent plus de 200 victimes) et, secondairement, de la Côte-d'Or, mal représentée dans le fichier initial, la progression des autres départements s'est réalisée de manière uniforme. Maintenant couverts, les secteurs initialement dépourvus de toute donnée comme les départements du Sud-Ouest (Landes, Gers, Lot-et-Garonne) restent bien épargnés par les attaques. On y trouve même encore deux départements sans information encore connue (Pyrénées-Atlantiques, Aude). Le Val de Loire (et en particulier les départements actuels de l'Indre-et-Loire et du Loir-et-Cher) reste largement en tête dans le nombre des victimes. Hormis la Corse - jamais concernée en raison de sa position insulaireet de trois départements méridionaux (mais ici le manque d'informations obère nos statistiques), l'ensemble du territoire national a été frappé par les attaques enregistrées.

En définitive, les constats initiaux demeurent. Dans cet espace s'opposent des îlots d'immunité relative (le nord du Bassin parisien, le Sud-Ouest, la Basse-Normandie) à des secteurs de forte concentration (Bourgogne, Champagne, Pays de la Loire, Nord de la Bretagne, Auvergne). Deux axes transparaissent nettement: un axe oriental nord-sud, de la Lorraine aux Alpes et à l'Auvergne, à travers le couloir de la Saône et du Rhône; un axe occidental dirigé est-ouest, du carrefour bourguignon à la pointe bretonne, en passant par le sud du Bassin parisien à travers le couloir ligérien. Au-delà des distorsions secondaires qui tiennent à l'inégale densité de notre information, la masse des données rassemblées révèle la forte présence de l'animal et les grandes zones à risque (carte 1). 


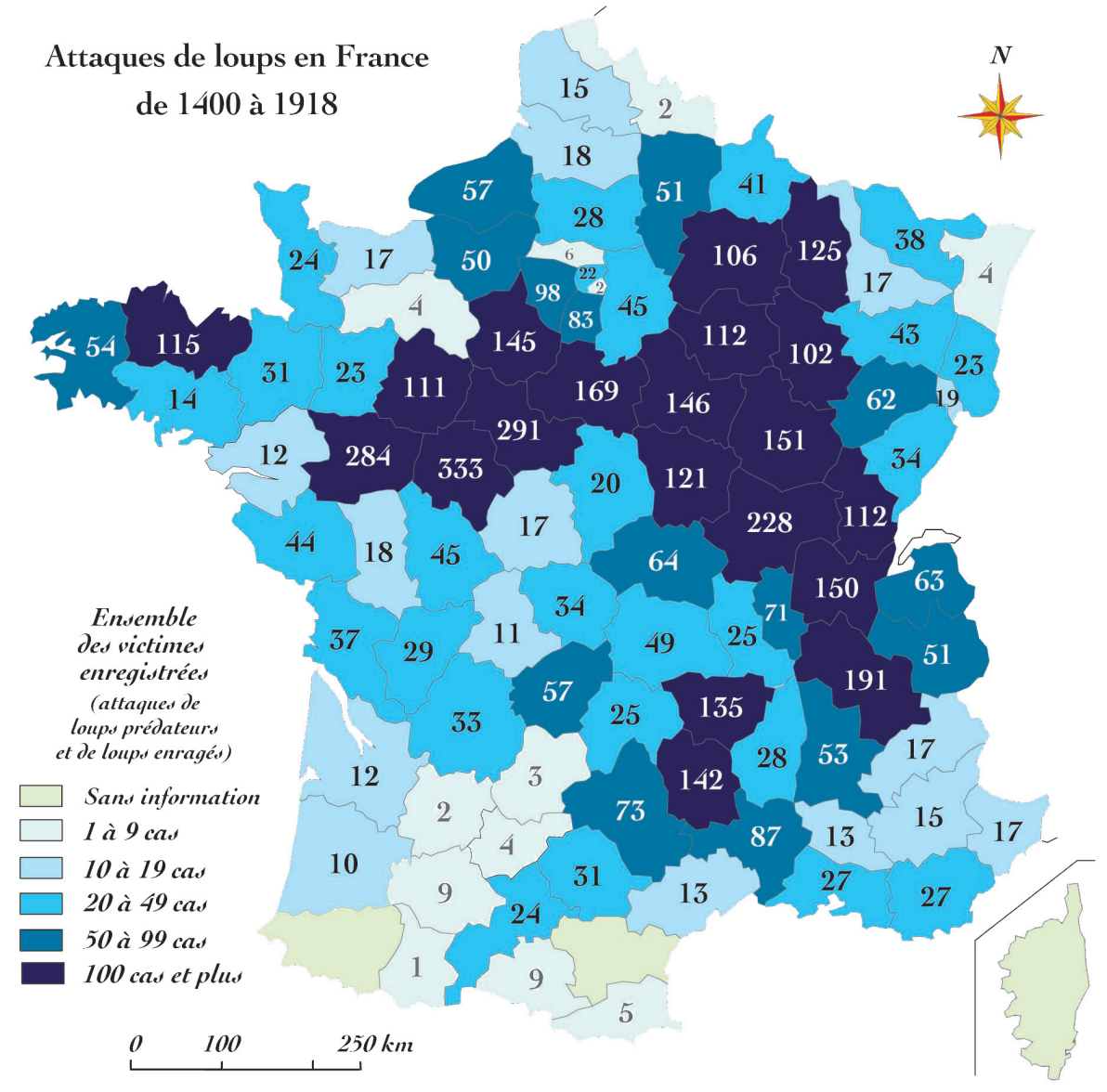

Carte 1 - Ensemble des victimes recensées: 5379 cas au 16 septembre 2010

Existe-il une relation entre la géographie des victimes humaines des loups et celle de la répartition de l'espèce? Une comparaison avec la densité des captures de loups à la fin du XVIII ${ }^{\mathrm{e}}$ siècle souligne une assez bonne correspondance entre fortes densités du prédateur et zones à risques (carte 2).

En l'an V et l'an VI de la République (1796-1798) les faibles prises que l'on observe dans le Sud-Ouest, la Basse-Normandie ou les régions du Nord forment le décalque des zones épargnées dans les attaques sur l'homme. Pour la Bourgogne, le Val de Loire ou le Nord de la Bretagne, on retrouve les mêmes fortes densités. La seule anomalie tient à l'Île-de-France 


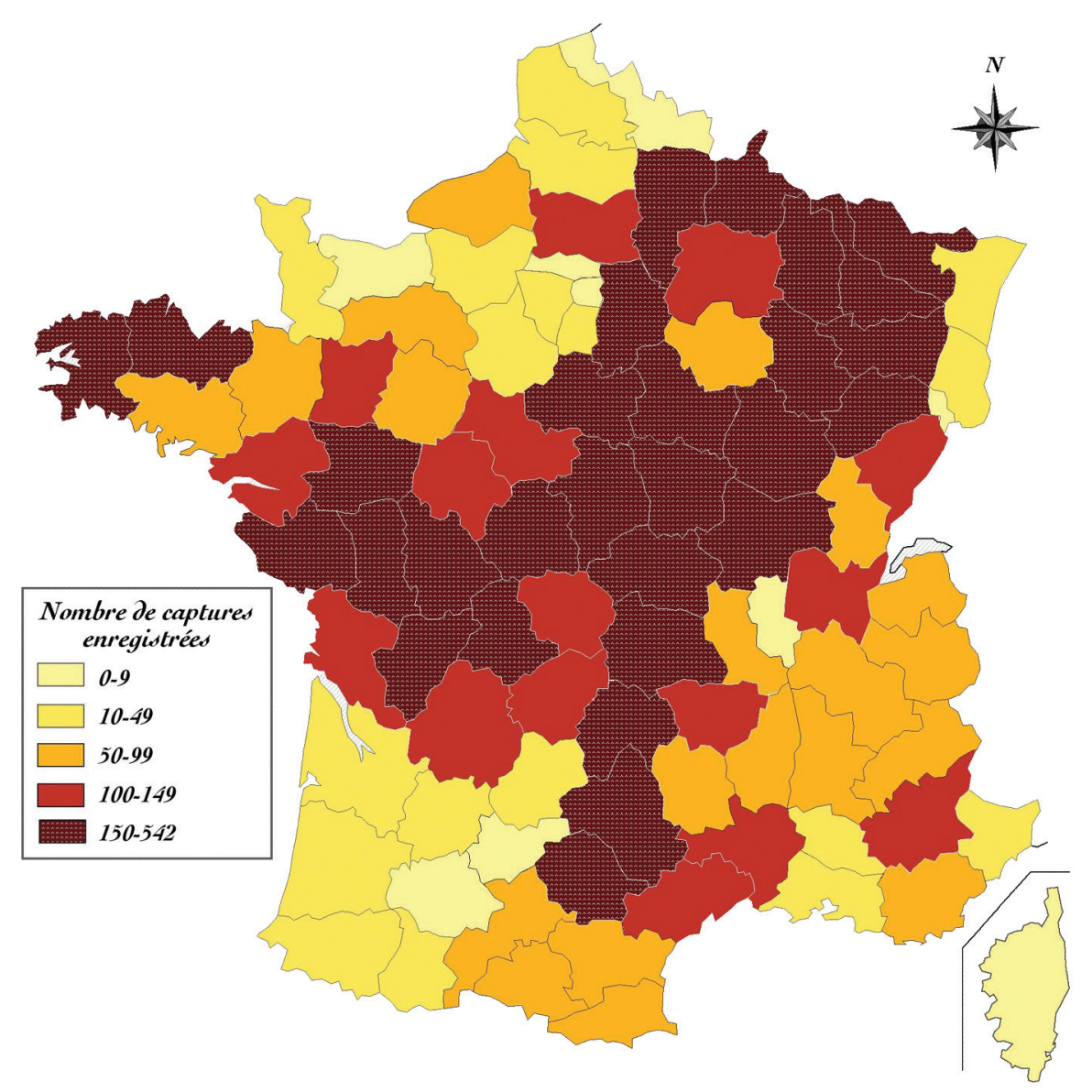

Carte 2 - Densité des loups à la fin du XVIII ${ }^{\mathrm{e}}$ siècle Évaluation par département à partir des captures de l'an V et de l'an VI

Source: François de Beaufort, Écologie historique du loup, 1988, p. 694.

(et secondairement à la Haute-Normandie), régions qui se sont débarrassées de la plupart de leurs loups entre la fin du XVII et à la fin du XVIII ${ }^{\mathrm{e}}$ siècle: or la carte générale des attaques y intègre les ravages qu'ils avaient commis au siècle précédent (départements actuels de l'Eure-et-Loir, des Yvelines et de l'Essonne). La distorsion tient donc à l'écart chronologique entre les deux cartes. Cette remarque faite, le parallélisme est frappant.

Si l'on compare maintenant la répartition départementale des victimes de loups prédateurs à celle des loups enragés, quelques divergences apparaissent (cartes 3 et 4 ). 


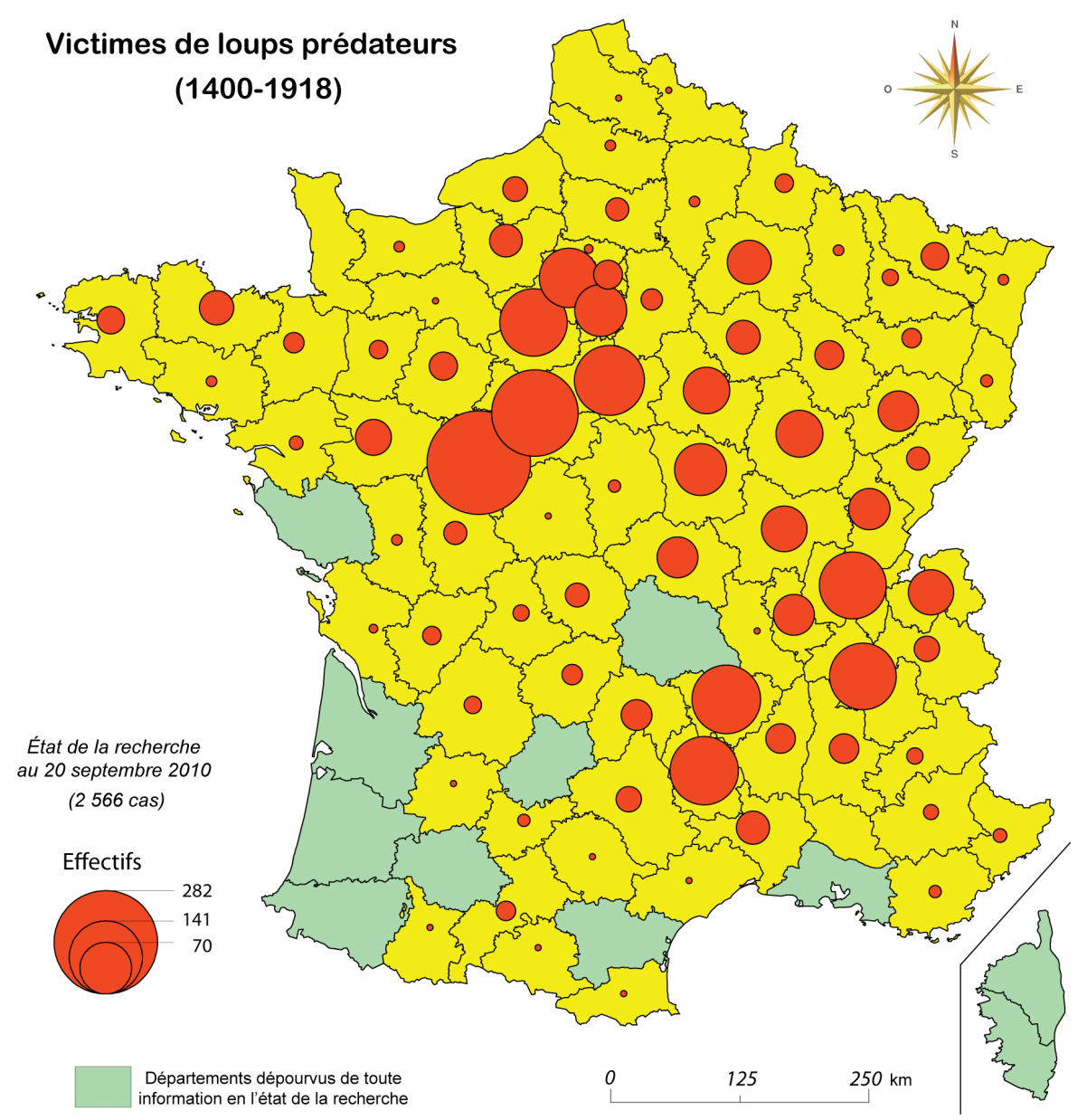

Carte 3 - Répartition des victimes de loups prédateurs (2 566 cas)

En dépit de la nature individuelle de la plupart des informations, les attaques de loups anthropophages paraissent davantage concentrées. De fait, dans 41 départements, on enregistre moins de 5 victimes de loups prédateurs et dans 52, moins de 10. Dans 16 départements (dont les deux départements corses) on ne décèle pour l'instant aucune victime. À l'inverse les 9 départements les plus frappés regroupent à eux seuls $56 \%$ des victimes de loups prédateurs (1 315 victimes sur 2 566): Indre-et-Loire (282 cas), Loir-et-Cher (197 cas), Ain (130 cas), Loiret (129 cas), Haute-Loire (124 cas), Lozère (123 cas), Eure-et-Loir (120 cas), Isère (117 cas) et Yvelines (93 cas) arrivent, en l'état 


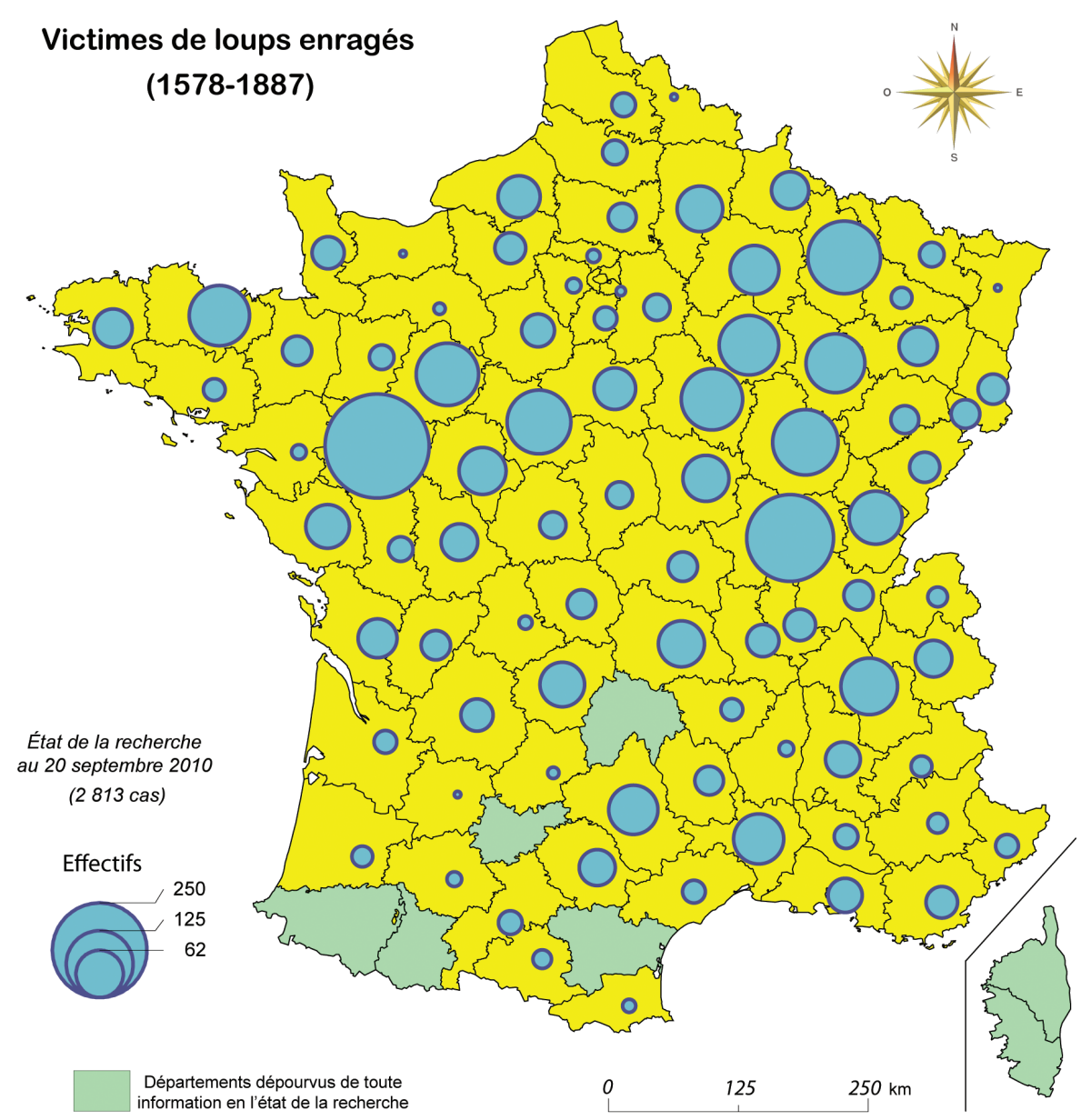

Carte 4 - Répartition des victimes de loups enragés (2 813 cas)

actuel de la recherche, en tête de classement. La majorité des victimes de loups anthropophages se localise dans un dixième du territoire français.

Si l'on opère le même calcul à propos des victimes de loups enragés, la réalité apparaît moins concentrée. On ne trouve que 20 départements à compter moins de 5 victimes et 23 seulement moins de 10 . Seuls 10 départements (dont les deux de la Corse) ne comprennent pour l'heure aucune donnée. Si l'on considère maintenant les départements qui arrivent en tête du nombre de victimes, les 9 premiers ne représentent que 38\% du total (1 082 sur 2 813) et il faut en rassembler 14 pour arriver à la moitié. Encore doit-on marquer, 
dans cette analyse, l'impact considérable des attaques de 1714 qui créditent le Maine-et-Loire de 244 victimes, contribuant à le faire passer en tête (250 cas), loin devant la Saône-et-Loire (174 cas) et la Meuse (122 cas). D'une manière générale, la répartition des attaques de loups enragés est plus régulière que celle des loups prédateurs. Déjà effectué en 2007, le constat se confirme. Si l'on réduit enfin les aléas liés à l'hétérogénéité des sources en ne considérant que les attaques indépendamment du nombre de victimes, la dispersion générale du risque est encore plus nette (carte 5).

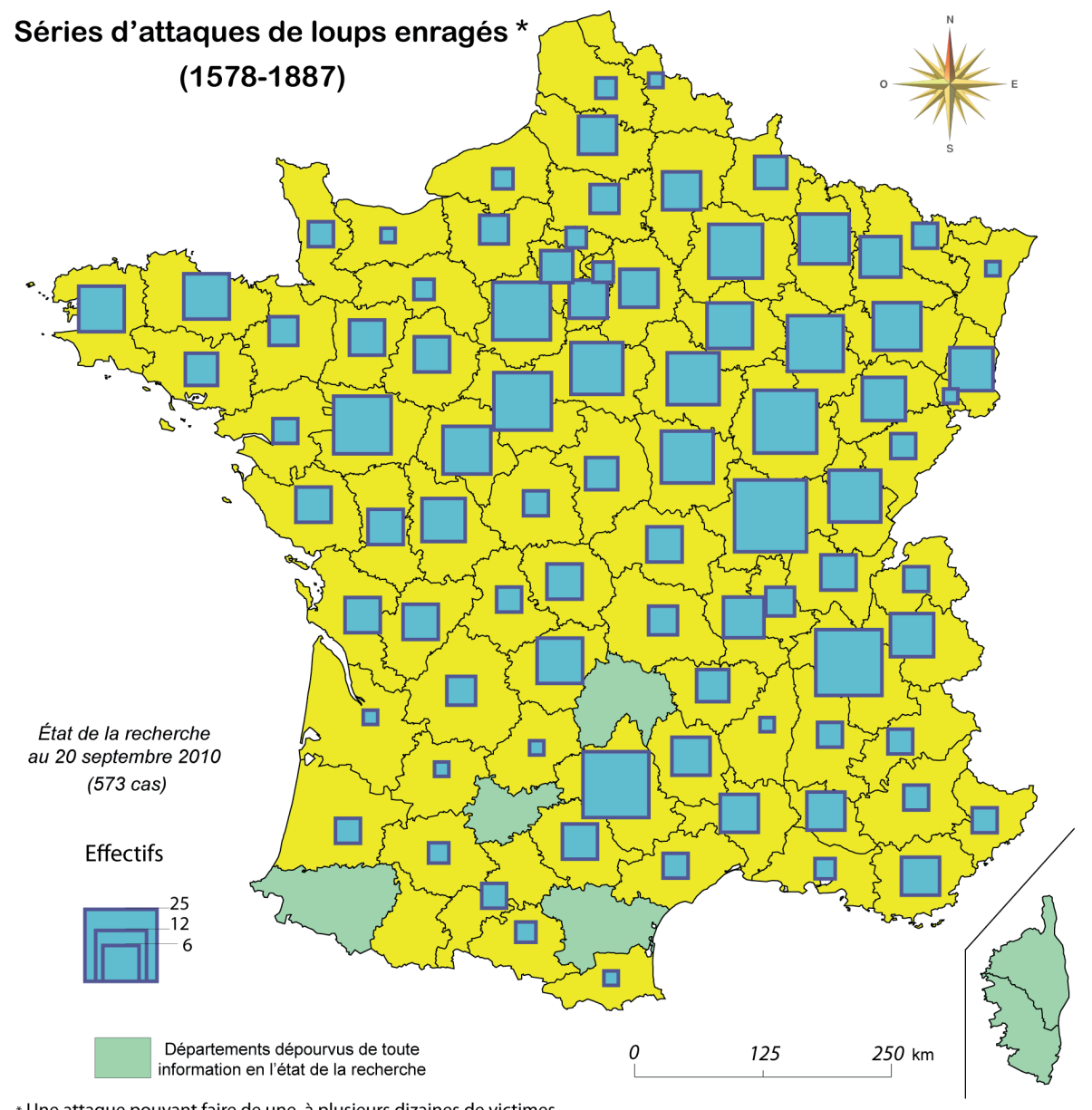

* Une attaque pouvant faire de une à plusieurs dizaines de victimes

Carte 5 - Séries d'attaques de loups enragés (573 cas) 
Cette dernière carte, qui corrige les biais introduits par l'inégalité de l'impact sur l'homme de chaque attaque de loup enragé, reflète encore mieux la répartition des densités de l'animal lui-même. En effet, maladie aléatoire, la rage est surtout fonction de l'importance des effectifs présents sur le territoire. À cet égard la comparaison avec la répartition des loups abattus vers 1800 (carte 2) souligne des correspondances étroites.

Réalisées à l'échelle nationale, ces cinq cartes n'ont qu'une vertu: offrir une vision d'ensemble de la présence du loup en France et signaler les zones à risques. Elles appellent à la fois des élargissements européens et, sur le territoire national, des observations plus fines, à partir d'analyses spatiales davantage localisées.

\section{Être attaqué par le loup: trois siècles d'évolution}

Sur près de trois siècles (1575-1870), l'abondance relative des informations enregistrées autorise une vue d'ensemble par périodes quinquennales. Au-delà des biais inévitables qui s'attachent à notre documentation, tout indique que s'opère une substitution de type d'agresseur au cours de cette longue période. Alors que les attaques de loups prédateurs paraissent l'emporter, et que les victimes de loups hydrophobes rester minoritaires jusqu'au règne de Louis XIV, le processus inverse s'amorce à partir de 1770 et, passé 1825 , seuls les loups enragés font la une des faits-divers. Cette tendance n'est pas sans conséquence dans l'image construite du «méchant loup». Résumons-en les grandes lignes (tableau 1).

Tableau 1 - Trois siècles d'attaques de loups sur l'homme Distribution chronologique des victimes dans le corpus de données rassemblé

\begin{tabular}{cccc}
\hline Périodes & $\begin{array}{c}\text { Victimes de } \\
\text { loups prédateurs }\end{array}$ & $\begin{array}{c}\text { Victime de } \\
\text { loups enragés }\end{array}$ & $\begin{array}{c}\text { Attaques de } \\
\text { loups enragés }\end{array}$ \\
\hline $1571-1575$ & 18 & - & - \\
\hline $1576-1580$ & 9 & 1 & 1 \\
\hline $1581-1585$ & 7 & 10 & 2 \\
\hline $1586-1590$ & 14 & 13 & 1 \\
\hline $1591-1595$ & 16 & - & - \\
\hline $1596-1600$ & 152 & - & - \\
\hline $1601-1605$ & 22 & - & - \\
\hline $1606-1610$ & 13 & - & 2 \\
\hline $1611-1615$ & 2 & 7 & 1 \\
\hline $1616-1620$ & 3 & 2 & \\
\hline
\end{tabular}




\begin{tabular}{|c|c|c|c|}
\hline Périodes & $\begin{array}{c}\text { Victimes de } \\
\text { loups prédateurs }\end{array}$ & $\begin{array}{c}\text { Victime de } \\
\text { loups enragés }\end{array}$ & $\begin{array}{c}\text { Attaques de } \\
\text { loups enragés }\end{array}$ \\
\hline $1621-1625$ & 3 & 3 & 3 \\
\hline $1626-1630$ & 2 & 14 & 2 \\
\hline $1631-1635$ & 56 & 10 & 6 \\
\hline $1636-1640$ & 50 & 0 & 0 \\
\hline $1641-1645$ & 8 & 7 & 4 \\
\hline $1646-1650$ & 19 & 12 & 5 \\
\hline $1651-1655$ & 91 & 11 & 4 \\
\hline $1656-1660$ & 12 & 10 & 5 \\
\hline $1661-1665$ & 25 & 10 & 2 \\
\hline $1666-1670$ & 11 & 43 & 12 \\
\hline $1671-1675$ & 28 & 6 & 4 \\
\hline $1676-1680$ & 65 & 10 & 6 \\
\hline $1681-1685$ & 63 & 17 & 6 \\
\hline $1686-1690$ & 25 & 1 & 1 \\
\hline $1691-1695$ & 262 & 26 & 6 \\
\hline $1696-1700$ & 84 & 4 & 3 \\
\hline $1701-1705$ & 7 & 15 & 5 \\
\hline $1706-1710$ & 52 & 43 & 10 \\
\hline $1711-1715$ & 172 & 246 & 13 \\
\hline $1716-1720$ & 61 & 135 & 9 \\
\hline $1721-1725$ & 5 & 49 & 20 \\
\hline $1726-1730$ & 31 & 116 & 24 \\
\hline $1731-1735$ & 57 & 21 & 11 \\
\hline $1736-1740$ & 24 & 146 & 19 \\
\hline $1741-1745$ & 99 & 9 & 7 \\
\hline $1746-1750$ & 151 & 83 & 19 \\
\hline $1751-1755$ & 138 & 34 & 15 \\
\hline $1756-1760$ & 63 & 30 & 12 \\
\hline $1761-1765$ & 151 & 109 & 16 \\
\hline $1766-1770$ & 68 & 106 & 24 \\
\hline $1771-1775$ & 9 & 101 & 26 \\
\hline $1776-1780$ & 17 & 72 & 15 \\
\hline $1781-1785$ & 9 & 81 & 12 \\
\hline $1786-1790$ & 11 & 42 & 12 \\
\hline $1791-1795$ & 7 & 61 & 10 \\
\hline $1796-1800$ & 40 & 228 & 54 \\
\hline $1801-1805$ & 38 & 185 & 43 \\
\hline
\end{tabular}




\begin{tabular}{cccc}
\hline Périodes & $\begin{array}{c}\text { Victimes de } \\
\text { loups prédateurs }\end{array}$ & $\begin{array}{c}\text { Victime de } \\
\text { loups enragés }\end{array}$ & $\begin{array}{c}\text { Attaques de } \\
\text { loups enragés }\end{array}$ \\
\hline $1806-1810$ & 33 & 67 & 15 \\
\hline $1811-1815$ & 96 & 181 & 28 \\
\hline $1816-1820$ & 66 & 103 & 20 \\
\hline $1821-1825$ & 10 & 86 & 13 \\
\hline $1826-1830$ & 2 & 60 & 16 \\
\hline $1831-1835$ & 3 & 31 & 9 \\
\hline $1836-1840$ & 0 & 36 & 5 \\
\hline $1841-1845$ & 4 & 3 & 2 \\
\hline $1846-1850$ & 4 & 27 & 3 \\
\hline $1851-1855$ & 1 & 51 & 2 \\
\hline $1856-1860$ & 3 & 6 & 3 \\
\hline $1861-1865$ & 2 & 2 & 8 \\
\hline $1866-1870$ & 0 & 5 & 3 \\
\hline $1871-1875$ & 1 & 21 & 2 \\
\hline $1876-1880$ & 1 & 9 & 1 \\
\hline $1881-1885$ & 0 & 10 & \\
\hline $1885-1890$ & 0 & & 2 \\
\hline
\end{tabular}

Pendant longtemps, Canis lupus a constitué un danger aussi bien comme animal enragé que comme prédateur. Pour les époques anciennes, il a sans doute dévoré davantage de proies humaines qu'il n'en a mordu comme animal enragé. Des périodes de «crises» surgissent: la période 1596-1600, à la fin des guerres de Religion, a constitué une phase dramatique (152 mentions se rattachent à ces cinq années), tout comme les années 1630 puis les années 1650 , au lendemain de la Fronde (91 occurrences de 1651 à 1656). Ces épisodes sont d'autant plus significatifs que les lacunes documentaires sont alors criantes: on ouvrait des actes de sépulture dans peu de paroisses et bien des registres ont disparu. Passé 1667, avec la mise en place d'une réglementation de l'état civil à l'échelle nationale, la base documentaire théorique s'améliore. C'est alors qu'on observe les pics les plus aigus: après l'épisode des attaques survenues autour de Versailles (1678-1683) c'est incontestablement entre 1691 et 1695 que se situe le paroxysme (262 cas enregistrés en l'état du fichier!). On comprend mieux alors l'écho que purent avoir à ce moment-là les Contes de ma mère l'Oye que publiait alors Charles Perrault. Les petits Poucets et les petits Chaperons rouges se comptaient chaque année par centaines. Ensuite la fin du règne de Louis XIV enregistre une recrudescence des agressions dans le quinquennat 1711-1715: rien ne manque alors au sombre tableau du royaume. 
Le long règne de Louis XV est marqué par l'épisode bien connu de la «Bête» du Gévaudan, qui contribue à hisser la barre dans le quinquennat 1761-1765 (151 victimes mais le chiffre comporte de simples blessés). Or cet épisode n'est pas plus grave, statistiquement que les séquences d'attaques intervenues autour de 1750 (151 occurrences de 1746 à 1750 et 138 encore de 1751 à 1755). Cependant, dans la longue cohorte des agressions, l'affaire du Gévaudan est la dernière du genre. Passé 1770 la chute est marquée et il faut attendre la seconde décennie du $\mathrm{XIX}^{\mathrm{e}}$ siècle pour voir resurgir, dans certaines régions, une menace qui s'était largement estompée jusqu'ici. De 1811 à 1820 se regroupent les dernières attaques importantes de loups prédateurs. Passé 1820 , le risque n'est plus qu'anecdotique. Il laisse la place à celui des loups enragés.

\section{Les grandes «bêtes»: des tueuses en série}

Or ces épisodes récurrents d'attaques de loups prédateurs tiennent une place particulière dans notre mémoire: ils correspondent à ce que les contemporains qualifiaient de «bêtes» tant l'assimilation au loup ordinaire, qui ne s'en prenait qu'au bétail, leur paraissait anthropologiquement impossible. De fait, les attaques en série qui endeuillaient à chaque fois une petite région pour quelques années, marquaient les esprits autant par l'atrocité de leurs effets que par la difficulté à éradiquer les agresseurs. De là cette «Bête du Gâtinais» qui, un siècle avant la celle du Gévaudan, fut tout aussi médiatisée. Le terme de «bête» intervient surtout lors des épisodes les plus dramatiques. C'est lui qui revient pour désigner les séquences les plus longues et les plus meurtrières de cette longue chronologie. À chaque fois que les morts (et les blessés) se comptaient en dizaines et que les agressions s'étendaient sur plusieurs mois, voire sur quelques années, la qualification de «bête» l'emportait dans le discours, sans occulter le loup pour autant. Au terme des attaques, lorsqu'on retrouvait l'agresseur - ou l'un d'entre eux - c'était toujours un grand loup, doté de qualités hors normes, sur lequel le rideau tombait. Encore fallait-il réussir la capture du prédateur. Lorsque le cas se produisait l'examen du prédateur permettait la démystification, et un «gros et puissant loup» apparaissait derrière la «bête». Ainsi en fut-il en 1634, pour la «bête furieuse» qui avait désolé les environs d'Évreux tout comme en 1655 pour la terrible «Bête du Gâtinais». À une échelle de ravages plus modeste, il en allait de même. En 1743, la «bête féroce» qui étrangla deux ou trois enfants en Haute-Bretagne était finalement «de forme d'un grand loup». La récupération du pied d'un enfant dans le ventre d'un loup à Chailly-en-Gâtinais en 1665 met fin à une psychose quand le public découvre que cette bête n'était qu'un «puissant loup» Le même travail de deuil 
s'observe en Touraine, lorsqu'on retrouve de la chair humaine dans les entrailles du dernier loup pourchassé en $1748{ }^{17}$. Dans chaque province concernée, le traumatisme des populations frappées par des attaques de loups en série forge l'image d'un coupable unique, la «bête anthropophage». Le constat n'est pas limité à la France puisque Mario Comincini l'avait déjà établi à propos de la «Bestia feroce» pour l'Italie du Nord ${ }^{18}$.

La répartition dans le temps des attaques fait apparaitre un lien récurrent avec les conflits armés, et en particulier la fin ou le lendemain des guerres civiles et étrangères qui multiplièrent les cadavres non enterrés. L'hypothèse d'un lien entre nécrophagie et anthropophagie que supposaient beaucoup de témoins trouve ici une confirmation. C'est très net pour la fin $\mathrm{du} \mathrm{XvI}^{\mathrm{e}}$ siècle (guerres de Religion) et pour les lendemains de la Fronde au milieu du XVII ${ }^{\mathrm{e}}$ siècle mais des cas restent possibles pour le règne personnel de Louis XIV et celui de Louis XV, des lendemains de la guerre de Hollande (1678) à ceux de la guerre de Sept Ans (1763).

En fait, la quasi-totalité des provinces françaises ont connu ce qui se produisait en Europe continentale, de l'Italie du Nord à la Russie. La mémoire de ces drames, transmise par les témoins des agressions ou par les blessés qui ont survécu - pour la plupart des enfants au moment des faits - n'a pu dépasser, sauf cas exceptionnels, le milieu du XIX ${ }^{\mathrm{e}}$ siècle. Étroitement localisées dans l'espace, les prédations du loup «mangeur d'hommes» étaient devenues de mauvais souvenirs dès la fin de l'Ancien Régime en dehors de quelques secteurs résiduels, comme les Cévennes ou la Bourgogne, qui subirent des attaques sporadiques jusque vers 1820. En revanche les attaques de loups enragés se sont maintenues plus longtemps: l'issue fatale de la maladie et les circonstances dramatiques qui s'attachent aux crises rabiques ont impressionné les contemporains jusqu'aux années 1880. La mémoire des agressions de loups atteints de la rage, qui ne faisaient point de distinction entre les âges, les sexes et les conditions sociales, s'est maintenue plus longtemps. Alors que pour sept décennies les loups disparaissent du territoire français, vers 1930, les enfants et les petits-enfants des victimes d'animaux enragés transmettent encore le souvenir de ces tragédies locales.

${ }^{17}$ Jean-Marc Moriceau, Histoire du méchant loup..., 2007, p. 82; Jean-Marc Moriceau et Philippe Madeline, éd., Repenser le sauvage..., 2010, p. 66 (document 11).

${ }^{18}$ Mario Comincini, L'Uomo e la «bestia antropofaga», 2002. 
Cependant, plus on s'éloigne de la réalité des événements, plus les risques de confusion et de déformation entre ces deux types de loups agresseurs se sont accrus, entre l'animal prédateur qui sélectionnait ses victimes, considérant occasionnellement l'homme comme une proie potentielle, et l'animal malade de la rage, qui n'était plus maître de son comportement. Dans un cas comme dans l'autre les conséquences pour les victimes terrifiaient leur entourage. Aux yeux de l'opinion, l'idée que l'homme pouvait en être «dévoré» restait ancrée: expression exacte dans le premier cas mais fausse dans pour le loup enragé qui, paralysé du pharynx, ne pouvait que lacérer et mordre cruellement ses victimes. Finalement l'image du «méchant loup» résulte un peu de cet amalgame. Alors que dans certains autres pays européens, comme le Portugal, l'Espagne ou la Roumanie, ou des espaces plus lointains, comme la Turquie, la Russie ou l'Inde, le loup prédateur faisait encore de furtives réapparitions, il avait déserté le terrain en France, victime d'un renversement complet du rapport de forces avec l'homme. C'est ce double héritage et ce décalage chronologique qui ont contribué à forger l'image si négative de Canus lupus à l'intérieur du territoire français. 